\title{
A rare disease with pregnancy: Castleman case report
}

\author{
Özen Esra Karaman*, Çetin Kılıççı, Pelin Özdemir Önder
}

1. Fenerbahçe University, Faculty of Health Sciences, Department of Midwifery, Istanbul/Turkey

2. Zeynep Kamil Woman and Child Diseases Education and Research Hospital, Gynecology and Obstetrics Clinic, Istanbul/Turkey

*Corresponding Author: Özen Esra Karaman

\begin{abstract}
Castleman's disease was first described by Castleman et al. in 1956 as a non-lymphoproliferative disease. ${ }^{1}$ Castleman's disease (CD), or angiofollicular lymphoid hyperplasia, is a rare disease with unknown etiology that can be easily misdiagnosed as lymphoma, neoplasm, or infection. Very few cases of pelvic origin and observed in pregnancy have been reported in the literature and are usually asymptomatic. Preoperative diagnosis is very difficult due to nonspecific imaging findings and rarity; most cases are diagnosed based on postoperative pathological examination. In this paper, a case of a 36-year-old pregnant woman suspected of adnexal origin in the uterine posterolateral, which was detected incidentally by ultrasound, was presented. The patient underwent a successful mass excision. Pathology of mass observed to be in the pelvic retroperitoneum was detected as localized unicentric and hyaline vascular CD. The study was conducted to discuss the diagnostic tools and perioperative management needed to identify the retroperitoneal unicentric Castleman case.
\end{abstract}

Keywords: Adnexal mass, pelvic tumor, pelvic pain, Castleman's disease, lymph node, pregnancy, hyaline, vascular

\section{Introduction}

$\mathrm{CD}$ is a rare benign lymphoproliferative disease. The patients are mostly asymptomatic, and the diagnosis is usually made incidentally. ${ }^{2}$ The etiology of the disease is still unknown. It can involve the lungs, pancreas, salivary glands, larynx, parotid, meninges, and extra lymphatic organs. Three histological variants, namely, hyaline vascular (HV), plasma cell, and mixed, have been described. While unicentric involvement generally shows HV histological features, most of the multicentric involvement is of plasma cell type. While unicentric involvement is generally observed in the mediastinum, pelvic involvement is rare. ${ }^{3}$

Since pelvic involvement often mimics adnexal solidheterogeneous masses, preoperative diagnosis is often difficult and can therefore be made postoperatively. ${ }^{4,5}$ On the other hand, $\mathrm{CD}$ has rarely been seen during pregnancy in the literature. Special immune conditions that develop during pregnancy can play a role in its development. In this article, a case of retroperitoneal CD, presenting as a localized HVtype giant lymph node hyperplasia, which is detected in rarely seen pregnancy, was presented. The study was conducted to report a case of $\mathrm{CD}$ in pregnancy and review the literature.

\section{Case Report}

During the routine health checks of a 36-year-old patient who was G3P1A1, was at her 18th gestational week, and didn't smoke or use alcohol, a $50 \mathrm{~mm}$ X $30 \mathrm{~mm}$ mass in the left adnexal lodge, which had a hypoechoic appearance and heterogeneous internal structure and which was observed to have blood build-up in color Doppler USG, was detected in the ultrasonography (USG) of the patient, who presented with a pain in the left inguinal region, which affected the left waist, and numbness in the left toes (Figure 1). On ultrasound, the ovaries could not be identified separately from the mass. No pathology was found in the physical examination of the patient. As the mass could not be diagnosed, the patient was admitted to the clinic for additional examination due to a suspected adnexal tumor. Hematological tests and blood and urine chemical values were all normal. Serological syphilis, herpes viruses, HIV and toxoplasma tests, antibodies were all negative. Tumor markers such as CA-125 showed no evidence of inflammation or malignancy. Anti EBV VCA IgC was positive, and Anti VCA IgM was negative. HHV-8 was not performed for EBV. The patient did not want to have CT and MRI scans due to pregnancy.

A laparotomy was planned for the patient due to a prediagnosis of adnexal mass in the $18^{\text {th }}$ gestational week for diagnosis and treatment purposes. During the operation, the uterus was in $18^{\text {th }}$-gestational week size, bilateral ovaries were found to be normal, and bilateral kidneys were palpated in a normal position. A retroperitoneal solid mass, approximately $6 \times 5 \mathrm{~cm}$ in size, was palpated on the left psoas muscle, close to the lower part of the left kidney, and medial to the external iliac artery. The retroperitoneal mass was loosened by opening the peritoneum and dissecting the surrounding tissues, and it was excised and sent to pathology. The mass was macroscopically $8 \times 6 \times 2.5 \mathrm{~cm}$ in size, with the section face being solid and having medium-hard consistency, in dirty-beige-pink color, and with some areas bleeding (Figure 2). A frozen section biopsy was performed and the result was reported as benign. On the postoperative $4^{\text {th }}$ day, the patient was discharged with full recovery. The mass was embedded in paraffin. It was administered CD2, CD3, CD15, and CD20. CD3 and CD20 had positive staining on immunohistochemistry. As a result of the histopathological examination, the final pathology was reported as HV-type $\mathrm{CD}$ (giant lymph node hyperplasia). Microscopic examination demonstrated a unicentric lymphoid follicle with a hyalinized germinal center in an onion-skin arrangement, which was consistent with the hyaline-vascular type of $\mathrm{CD}$. On followup examination, the patient was sent to a hematology clinic for detailed evaluation and treatment if needed. So she did not receive any further treatment, and the follow-up is going on currently. The patient had an uneventful postoperative course, and she gave birth spontaneously in the 40th 


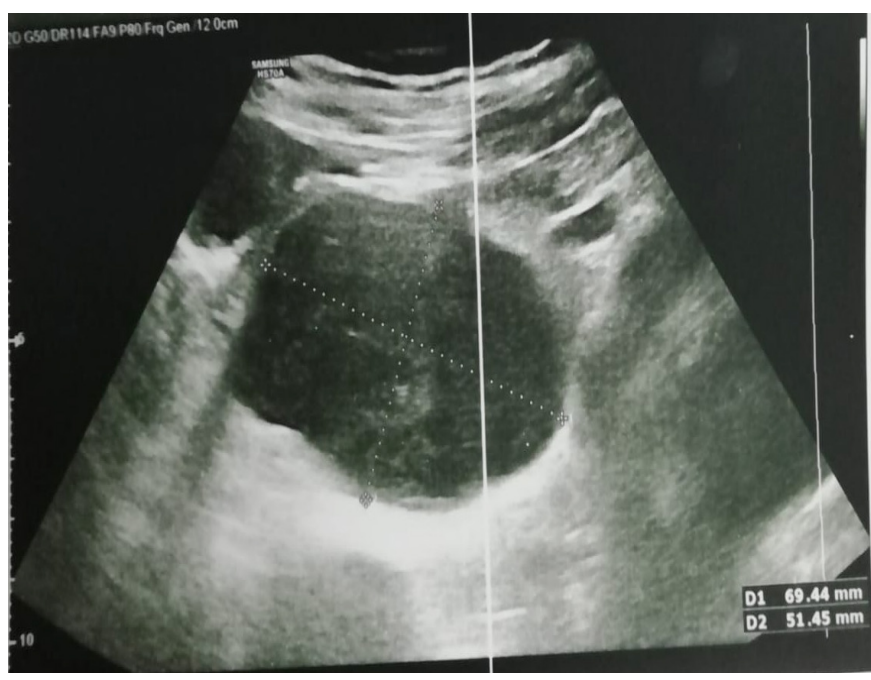

Figure 1. Preoperative ultrasonography image

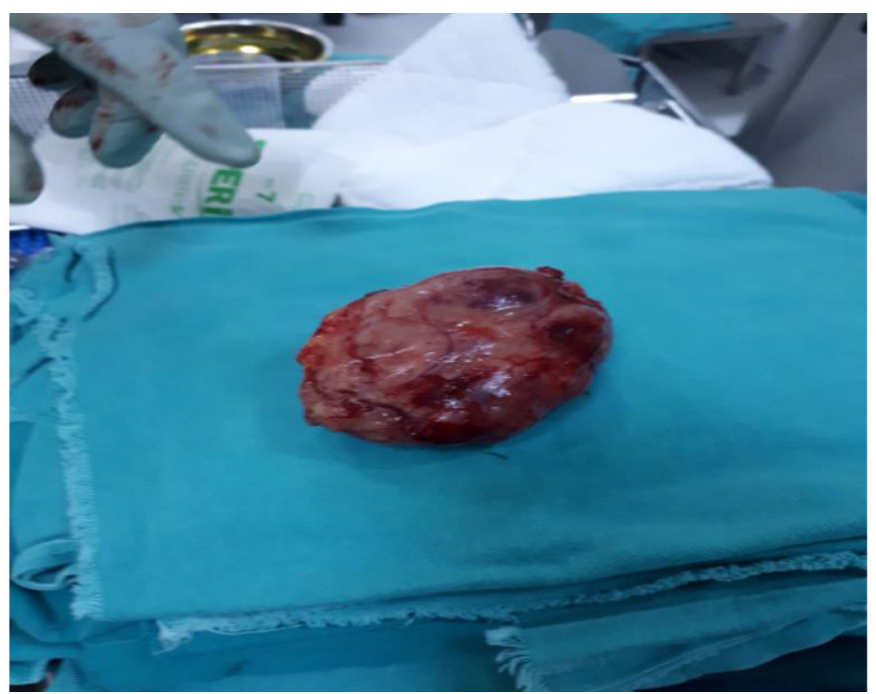

Figure 2. Excision Material

gestational week. The baby, who was a girl and weighed 3280 $\mathrm{g}$, was in good condition and was discharged the next day.

The case was asymptomatic after two years of follow-up, and no recurrence was observed.

For the clinical sample used in the study, human well-being and ethical rules were followed, and the consent of the patient was obtained.

\section{Discussion}

CD or angiofollicular lymphoid hyperplasia is a rare member of the heterogeneous group of lymphoproliferative diseases, which was first described by Dr. Benjamin Castleman in 1956. Its detailed pathophysiology is still unknown, and possible areas of its occurrence have been reported as the mediastinum $(63 \%)$, abdomen $(11 \%)$, and retroperitoneal area $(7 \%)$, and axilla $(4 \%){ }^{6}$

$\mathrm{CD}$ is divided into three subtypes: hyaline vascular type (HV), plasma cell type (PC), and a combination of HV and PC types (Mixed). ${ }^{7} \mathrm{HV}$ shows follicular dendritic cell prominence, dysplasia, and hypervascularity in the interfollicular regions. The PC type, on the other hand, shows an increased number of follicles with large, hyperplastic germinal centers. Kawamura et al. reported that of the 132 retroperitoneal CD cases, $87 \%$ were HV type, $8 \%$ were PC type, and 5\% were the mixed type. ${ }^{8}$

$\mathrm{CD}$ is classified as unicentric Castleman disease (UCD) or multicentric Castleman disease (MCD) according to its distribution. UCD is usually of the HV type, is asymptomatic, and does not yield abnormal laboratory findings, and most cases are diagnosed incidentally. While not associated with HIV or HHV-8 infection, it is often seen in the third or fourth decade. ${ }^{9}$ In contrast, MCD is mostly of the plasma cell type, often occurring at an early age in the $6^{\text {th }}$ decade. In addition to other findings, it may also indicate coexistence with HIV and HHV-8 infection, enlargement of multiple lymph nodes, fever, fatigue, weight loss, anemia, decreased platelets, increased CRP, hyper $\gamma$ globulin, and hyper serum IL-6. These findings are associated with the histological type, and while $90 \%$ of UCD cases are HV type, almost all MCD cases are PC type. ${ }^{10}$ In our case, the mass showing retroperitoneal involvement indicated HV type histologically, and it was diagnosed to be UCD because no other lesions were observed.

In the pelvic CD diagnostic approach, imaging techniques, such as USG, CT, or MRI, reveal the characteristics of a vascular mass. In ultrasound, the $C D$ is usually imaged homogeneously and resembles lymphoma in the differential diagnosis. ${ }^{4}$ It has been reported that the disease shows hypervascularity when evaluated with color Doppler USG. This was the instance in our case. In the presence of a hypervascular, homogeneous hypoechoic pelvic mass on USG imaging, the CD should be considered in the differential diagnosis.

In the localized form, patients are generally asymptomatic and are diagnosed incidentally after routine examination, chest radiography, and abdominal USG. In the case of localized $\mathrm{CD}$, the size of the mass varies between 1 and $12 \mathrm{~cm}$ and may cause pain due to the pressure effect. In addition, although it generally has an asymptomatic course, surgical resection is enough for treatment. In our case, who presented with the complaints of pain in the left inguinal region and numbness in the foot, a retroperitoneal pelvic localized mass with hypervascular, homogeneous, and hypoechoic characteristics was detected while the USG was being taken, and a full cure was achieved after surgical excision. No recurrence was observed during the one-year follow-up.

MCD patients always show symptoms due to increased IL-6 levels (asthenia, fever, weight loss, anemia, and thrombocytopenia). Peripheral lymphadenopathy and hepatosplenomegaly are frequently detected. Unlike the localized form, there is no consensus yet on the treatment of MCD. Chemotherapy and corticotherapy are administered in addition to the surgical operation. The response is variable, and the prognosis is poor in these treatments.

Pelvic localized CD may indicate clinical findings similar to clinical characteristics of tubo-ovarian abscess, endometriotic cysts, or mature cystic teratoma. Although the incidence of $\mathrm{CD}$ with pelvic involvement is low, it can be difficult to diagnose $C D$ at initial evaluation due to the clinical similarities of other pelvic masses and may be included in the differential diagnosis of a pelvic mass. Considering that $80 \%$ of primary retroperitoneal masses are malignant, surgical excision of the mass and pathological diagnosis are important in differential diagnosis. $^{11}$

The CD that is detected during pregnancy in the literature is limited to a few cases. In the case reported by Tsukamoto et al. in 1979, in the follow-up of the pelvic mass detected during the routine antenatal examination in the 8th gestational week, the $12 \times 10 \mathrm{~cm}$-mass was removed in the 41 st week of gestation by excisional surgery during cesarean section because the labor did not progress due to the 
pressure of the mass on the birth canal, and a perioperative massive blood transfusion was needed due to hypogastric artery vascularization. The pathology result of the mass was reported as giant vascular lymphoid benign hyperplasia. ${ }^{12}$

The pathology of the mass detected in an asymptomatic patient in the $15^{\text {th }}$ gestational week, reported by Yasuda et al (1987), which was administered a laparotomy excision, was HV CD. ${ }^{13}$

The $82 \times 150 \mathrm{~mm}$ mass, which was detected in the routine USG examination of a 21 -year-old pregnant patient by Baser et al. (1989) and was preliminarily diagnosed as adnexal, was administered a laparotomic excision in the $19^{\text {th }}$ gestational week. It was a retroperitoneal HV-type CD. It was reported that the patient had no additional complaints and laboratory findings other than rare pain attacks in the lower abdomen, but that it bled $2000 \mathrm{ml}$ due to the vascular nature and the difficulty of excision. ${ }^{14}$

The mass reported by Abramov et al. (1997), which was $10 \times 8 \mathrm{~cm}$ in size, was adjacent to the lower pole of the retroperitoneal right kidney and was detected during the examination of a patient who presented with bleeding in the second trimester of pregnancy, was diagnosed as HV CD as a result of the laparotomy excision. ${ }^{15}$ In our case, like other cases in the literature, laparotomy excision was performed in the second trimester of pregnancy due to suspicion of a pelvic tumor. Like all pregnant cases, a complete cure was achieved with surgical resection, and no recurrence was observed during follow-ups.

Castleman disease can adversely affect pregnancy by increasing stressors and can even cause pregnancy loss.

Treatments, such as chemotherapy, radiotherapy, immunotherapy, and antiretroviral drugs, do not cover patients with pregnancy in clinical management, and the surgical management of the mass is difficult in terms of bleeding due to vascularization in women with pregnancy. However, in conclusion, it should be noted that surgery is the treatment of choice, regardless of pregnancy status. Antiretroviral drugs are never indicated for unicentric Castleman disease. Radiation and chemotherapy is sometimes used in unresectable cases to shrink the tumor enough to resect, but this is not appropriate during pregnancy. Surgical resection is recommended for any type of CD and is required for differential diagnosis. It has been reported that HV-type CD does not have negative consequences after surgical resection and that it rarely recurs. This was the same in our case, and there was no recurrence one year after the surgical resection. However, careful observation is required due to its prevalence during pregnancy and the rarity of retroperitoneal cases. Based on the current findings, preoperative diagnosis of $\mathrm{CD}$ was difficult. In conclusion, the $\mathrm{CD}$ should be included in the preliminary diagnoses in pelvic/retroperitoneal localized masses. Excellent early and late outcomes are obtained with complete surgical resection.

\section{Ethical Statement}

Consent was obtained from the patient.

\section{Conflict of Interest}

No conflict of interest was declared by the authors.

\section{Financial Disclosure}

No financial support was declared by the authors.

\section{Authorship Contributions}

All authors contributed equally while preparing this study.

\section{References}

1. Castleman B, Iverson L, Menendez VP. Localized mediastinal lymphnode hyperplasia resembling thymoma. Cancer. 1956 Jul-Aug; 9(4):822-30. doi: 10.1002/1097-0142(195607/08)9:4<822::aidcncr2820090430>3.0.co;2-4. PMID: 13356266.

2. Karapolat S, Onen A, Sanli A, Eyuboglu M. Giant solitary fibrous tumor of the pleura. Lung. 2008 Jul-Aug; 186(4):269-70. doi: 10.1007/ s00408-008-9083-9. PMID: 18357491.

3. Gopi P, Potty VS, Kaurav RS, Govindan K. Unicentric Castleman's Disease as a Localized Retroperitoneal Mass: A Case Report and Review of Literature. Int J Appl Basic Med Res. 2018; 8(4):259-62. doi:10.4103/ijabmr.IJABMR_256_17.

4. Hsieh C-H, Changchien C-C, Lan K-C, Huang C-C, Shen C-C, Chang S-Y, et al. Pelvic Castleman's disease presenting as an adnexal tumor. Acta Obstet Gynecol Scand. 2004 Mar; 83(3):311-3. doi: 10.1111/j.0001-6349.2004.0089a.x. PMID: 14995930.

5. Karaman E, Ateş Ç, Kolusarı A, Alkış İ, Şahin HG, Gül A, et al. A rare case of pelvic Castleman's disease mimicking an adnexal tumor, East J Med 23(4): 344-346, 2018 DOI: 10.5505/ejm.2018.61580.

6. Bucher P, Chassot G, Zufferey G, Ris F, Huber O, Morel P. Surgical management of abdominal and retroperitoneal Castleman's disease. World J Surg Oncol. 2005 Jun 7;(3): 33. doi: 10.1186/1477-7819-3-33. PMID: 15941478; PMCID: PMC1166581.

7. Keller AR, Hochholzer L, Castleman B. Hyaline-vascular and plasma-cell types of giant lymph node hyperplasia of the mediastinum and other locations. Cancer. 1972 Mar; 29(3):670-83. doi: 10.1002/1097-0142(197203)29:3<670::aidcncr2820290321>3.0.co;2-\#. PMID: 4551306.

8. Nakamura Y, Tokuyama O, Muso A, Kawamura N, Yasui T, Ishiko O. Asymptomatic pelvic Castleman disease in an infertile woman: case report. Arch Gynecol Obstet. 2004 Jan; 269(2):156-8. doi: 10.1007/ s00404-002-0420-6.

9. Soumerai JD, Sohani AR, Abramson JS. Diagnosis and management of Castleman disease. Cancer Control J Moffitt Cancer Cent. 2014 Oct; 21(4):266-78. doi: 10.1177/107327481402100403. PMID: 25310208.

10. MacDonald SR, Lurain JR, Hoff F, Variakojis D, Fishman DA. Castleman disease presenting as a pelvic mass. Obstet Gynecol. 1996 May; 87(5 Pt 2):875-7. PMID: 8677123.

11. Takihara H, Yamakawa G, Baba Y, Takahashi M, Ishihara T. Castleman disease. Unusual retroperitoneal location indistinguishable from malignant tumor in preoperative angiographic appearance. Urology. 1993 Feb; 41(2):162-4. doi: 10.1016/0090-4295(93)90173-8. PMID: 8497992

12. Tsukamoto N, Iraha H, Matsuyama T, Wentz WB. Giant lymph node hyperplasia. Gynecol Oncol. 1980 Jun; 9(3):394-404. doi: 10.1016/0090-8258(80)90051-7. PMID: 7380351.

13. Yasuda J, Sawada S, Tomioka M, Yamamoto T, Okada H. Castleman's Disease Associated with Pregnancy: A Case Report. Asia Oceania J Obstet Gynaecol. 1987 Dec;13(4):451-4. doi: 10.1111/j.14470756.1987.tb00290.x. PMID: 3322243.

14. Başer İ, Dilek S, Pabuçcu R, Ülgenalp İ, Mermut S, Finci R. Unexpected Nature of a Pelvic Mass in Pregnancy: Castleman's Disease. Gynecol Obstet Invest. 1989; 28(1):53-5. doi: 10.1159/000293507. PMID: 2673950.

15. Abramov Y, Nadjari M, Abrahamov A, Reinus C, Anteby S. Castleman disease in pregnancy. Obstet Gynecol. 1997 Oct; $90(4 \mathrm{Pt}$ 2):653-4. doi: 10.1016/s0029-7844(97)00222-6. PMID: 11770581. 